\title{
"Superluminal" scalar fields and black holes
}

\author{
Eugeny Babichev* \\ Laboratori Nazionali del Gran Sasso, INFN \\ E-mail: babichev@lngs.infn.it
}

\section{Viatcheslav Mukhanov}

Arnold-Sommerfeld-Center for Theoretical Physics, Ludwig-Maximilians-Universität München, Germany

E-mail: mukhanov@theorie.physik.uni-muenchen.de

\author{
Alexander Vikman \\ Arnold-Sommerfeld-Center for Theoretical Physics, Ludwig-Maximilians-Universität München, \\ Germany \\ E-mail: vikman@theorie.physik.uni-muenchen.de
}

\begin{abstract}
Non-canonical scalar field theories may lead to superluminal propagation of perturbations in nontrivial backgrounds. In spite of the superluminal propagation the causal paradoxes do not arise in these theories and in this respect they are not less safe than General Relativity. Here we consider the accretion of non-canonical k-essence-like scalar fields onto a Schwarzschild black hole, and we show that one can send information from inside a black hole. This information is encoded in perturbations of the field propagating in non-trivial scalar field backgrounds, which serves as a "new ether".
\end{abstract}

From Quantum to Emergent Gravity: Theory and Phenomenology

June 11-15 2007

Trieste, Italy

${ }^{*}$ Speaker. 


\section{Introduction}

Field theories with nonstandard kinetic terms have been subjects of investigation since a long time ago [1]. In cosmology they were first introduced in the context of k-inflation and k-essence models [2]. Due to non-linearity of the equations of motion, small perturbations propagate in a new, effective, metric which may differ from the gravitational one ${ }^{1}$. In other words, a background dynamically breaks Lorentz invariance and serves as a "new ether". In this case, depending on a model and a background solution, the propagation speed of perturbations can be greater or less than the speed of light.

In literature there is a number of examples of theories allowing superluminal propagation of signals. Successful cosmological k-essence models seems to require a superluminal sound speed during a period of cosmic evolution $[4,5]^{2}$. In the Einstein-Aether theory some modes can propagate with superluminal velocities [8].

As it was argued in [9], in spite of the superluminal propagation the causal paradoxes do not arise in k-essence-like theories and in this respect they are not less safe than General Relativity (GR).

In our previous work [10] we have shown that it is principally possible to send information from the interior of a black hole (BH) without modifying Hilbert-Einstein action ${ }^{3}$. This may happen provided a special type of scalar field theory with noncanonical kinetic term is realized in nature. In our approach we have considered the accretion of a particular noncanonical field $\phi$ onto a black hole ${ }^{4}$. The scalar field flow forms an acoustic black hole similar to the well known "dumb hole" [14].

Here we review our results from the paper [10] with the main stress on the issues of causality and acoustic metric in eikonal approximation.

\section{Model}

In our paper [10] we considered a scalar field $\phi$ with the generally covariant and Lorentz invariant action

$$
S=\int \mathrm{d}^{4} x \sqrt{-g} p(X), \text { where } p(X)=\alpha^{2}\left[\sqrt{1+\frac{2 X}{\alpha^{2}}}-1\right] .
$$

The Lagrangian $p(X)$ depends only on $X \equiv \frac{1}{2} \nabla_{\mu} \phi \nabla^{\mu} \phi$, and $\alpha$ is a free parameter of the theory. Throughout the paper $\nabla_{\mu}$ denotes the covariant derivative and we use natural units in which $G=$ $\hbar=c=1$. The kinetic part of the action is the same as in [7] and for small derivatives, that is, in the limit $2 X \ll \alpha^{2}$, it describes the usual massless free scalar field. In the case of arbitrary $p(X)$ the equation of motion for $\phi$ is

$$
G^{\mu v} \nabla_{\mu} \nabla_{v} \phi=0, \text { where the induced metric } G^{\mu v} \equiv g^{\mu v}+\frac{p_{, X X}}{p_{, X}} \nabla^{\mu} \phi \nabla^{v} \phi,
$$

\footnotetext{
${ }^{1}$ Another possibility to obtain the propagation cone different from the gravitational one is to consider two kinetically coupled to scalar fields [3].

${ }^{2}$ The models with superluminal sound speed may have other interesting applications in cosmology [6, 7].

${ }^{3}$ In bimetric theories this possibility can be easily achieved [11].

${ }^{4}$ The accretion of other noncanonical scalar fields were studied in e.g. [12, 13].
} 
and $p_{, X} \equiv \partial p / \partial X$. Throughout the paper we assume the condition $p_{, X}>0$ to be true, which is obviously satisfied for the concrete Lagrangian (2.1). The equation (2.2) is hyperbolic and its solutions are stable with respect to high frequency perturbations provided $\left(1+2 X p_{, X X} / p_{, X}\right)>0$ $[6,16,17,18]$. Propagation vectors $N^{\mu}$ are tangent to characteristic surface and define the influence cone:

$$
G_{\mu \nu}^{-1} N^{\mu} N^{v}=0, \text { where } G_{\mu \nu}^{-1}=g_{\mu \nu}-\frac{p_{, X X}}{p_{, X}+2 X p_{, X X}} \nabla_{\mu} \phi \nabla_{v} \phi
$$

is inverse matrix to $G^{\mu \nu}$. The influence cone is larger than the light cone if $p_{, X X}<0[16,17,18]$. In this case the front of small perturbations of $\phi$ propagates faster than light. If a background $\phi(x)$ is trivial, $\nabla_{\mu} \phi=0$, then perturbations (small discontinuities) propagate with the speed of light. Therefore only nontrivial backgrounds $\phi(x)$ spontaneously break the Lorentz invariance. Despite the fact that the action (2.1) is manifestly Lorentz invariant the action for perturbations $\delta \phi$ around a non-trivial background solution is only generally covariant but not Lorentz invariant anymore. This background can be considered as a medium or "new ether". Observers moving differently with respect to this medium may disagree in the results of some measurements. Moreover in the case of superluminal propagation there is no Lorentz invariant notion of causality $[18,15]$. However, by virtue of the hyperbolicity of the system even in this case there may exist some Cauchy hypersurfaces [17] and therefore observers for which the causality is well defined [19]. Nevertheless, there are backgrounds [15] where closed time like curves (CTC) exist. However, in the standard GR [20] it is also the case. The so-called chronology protection conjecture [21] may preclude the existence of CTC. For a more detailed discussion of causality in the theories with spontaneously broken Lorentz-invariance see paper the [9]. For the energy-momentum tensor we have $T_{\mu \nu}=p_{, X} \nabla_{\mu} \phi \nabla_{\nu} \phi-p g_{\mu \nu}$. Thus the Null Energy Condition $T_{\mu \nu} n^{\mu} n^{v} \geq 0{ }^{5}$ is satisfied if $p_{, X} \geq 0$. This is always the case for our model (2.1) and hence the black hole area theorem [22] holds. It is well known that, if $\nabla_{\nu} \phi$ is timelike (that is, $X>0$ in our convention), then the system with general $p(X)$ is formally equivalent to a perfect fluid with the pressure $p=p(X)$, energy density $\varepsilon(X)=2 X p_{, X}(X)-p(X)$, the four-velocity $u_{\mu}=\nabla_{\mu} \phi / \sqrt{2 X}$ and the sound speed $c_{s}^{2} \equiv \partial p / \partial \varepsilon=p_{, X} / \varepsilon_{, X}$. Specializing to the case of the Lagrangian (2.1) we have

$$
c_{s}^{2}=1+\frac{2 X}{\alpha^{2}} \geq 1, \frac{\varepsilon}{\alpha^{2}}=\left(1-c_{s}^{-1}\right), \frac{p}{\alpha^{2}}=\left(c_{s}-1\right), G_{\mu \nu}^{-1}=g_{\mu v}+\frac{\nabla_{\mu} \phi \nabla_{\nu} \phi}{\alpha^{2}} .
$$

\section{The background solution and propagation of small perturbations}

Here we sketch how to find a stationary spherically symmetric background solution for the scalar field falling onto a Schwarzschild black hole. In the Eddington-Finkelstein coordinates ${ }^{6}$ the metric takes the form:

$$
\mathrm{d} s^{2}=f(r) \mathrm{d} V^{2}-2 \mathrm{~d} V \mathrm{~d} r-r^{2} \mathrm{~d} \Omega^{2}, \text { where } f(r) \equiv 1-\frac{r_{g}}{r}, \quad r_{g} \equiv 2 M .
$$

In [10] we verified that there is a broad range of free parameter $\alpha^{2}$ for which the infalling field has a negligible influence on the black hole, that is, we consider the accretion of a test fluid in a given

\footnotetext{
${ }^{5} n^{\mu}$ is null vector in $g_{\mu v}$.

${ }^{6}$ Note that these coordinates are regular at Schwarzschild horizon.
} 
gravitational field. The stationarity and cosmological boundary conditions at spatial infinity imply the following ansatz for the solution:

$$
\phi(V, r)=\alpha \sqrt{c_{i}^{2}-1}\left(V+\int^{r} F\left(r^{\prime}\right) d r^{\prime}\right), \text { where } c_{i} \text { is the speed of sound at infinity. }
$$

For every solution $F(r)$ the induced acoustic line element (in eikonal approximation) is

$$
\mathrm{d} S^{2} \equiv G_{\mu \nu}^{-1} \mathrm{~d} x^{\mu} \mathrm{d} x^{\nu}=\left(c_{i}^{2}-\frac{r_{g}}{r}\right) \mathrm{d} V^{2}-2 \mathrm{~d} V \mathrm{~d} r\left(1-\left(c_{i}^{2}-1\right) F\right)+\left(c_{i}^{2}-1\right) F^{2} \mathrm{~d} r^{2}-r^{2} \mathrm{~d} \Omega^{2} .
$$

In this acoustic metric the coordinate $V$ is timelike. Therefore from (3.3) it follows that there exists sonic horizon at $r_{\star}=r_{g} / c_{i}^{2} \leq r_{g}$. Substituting (3.2) into (2.2) and (3.3) one can obtain that the only physical solution which satisfies all boundary conditions and for which the acoustic space-time (3.3) is not singular for $r \geq r_{\star}$, is given by:

$$
F(x)=\frac{1}{f(x)}\left(\sqrt{\frac{c_{i}^{2}+f(x)-1}{f(x) x^{4} c_{i}^{8}+\left(c_{i}^{2}-1\right)}}-1\right), \text { where } x \equiv r / r_{g} .
$$

And for the sound speed one obtains $c_{s}^{2}(x)=x^{3} c_{i}^{8} /\left[1+c_{i}^{2}(x-1)\left(1+x c_{i}^{2}+x^{2} c_{i}^{4}\right)\right]$. The acoustic spacetime (3.3) with the function (3.4) describes an analogue black hole with the horizon which is inside the Schwarzschild horizon. Therefore it is possible to use perturbations $\delta \phi$ around this background (3.2),(3.4) as signals and to send information from the region $r_{\star}<r<r_{g}$ between two horizons, see Fig. 2 from [10]. On the background solution $\nabla^{\mu} \phi$ is a time like non-vanishing vector field well defined for $r \geq r_{\star}$. Thus in accordance with [23] the acoustic space time is stably (and therefore strongly) causal for $r \geq r_{\star}$. Suppose that a spacecraft moves together with the falling background field and sends the acoustic signals with the frequency $\omega_{e m}$. After simple calculations one can obtain that an observer at rest at the spatial infinity will detect these signals at the frequency $\omega_{\text {inf }}$ :

$$
\frac{\omega_{\text {inf }}}{\omega_{\text {em }}}=\left(1-\left(\frac{r_{\star}}{r}\right)^{2}\right) \sqrt{\frac{1-r_{g} / r}{1-c_{s}^{2}(r)\left(r_{\star} / r\right)^{4}}}
$$

This expression corrects our result from [10]. Note that the ratio $\omega_{e m} / \omega_{\text {inf }}$ is finite for any $r>r_{\star}$ and it vanishes for $r=r_{\star}$. In particular for the moment of crossing the Schwarzschild horizon we have $\omega_{\text {em }} / \omega_{\text {inf }}=c_{i}^{4} \sqrt{1+c_{i}^{2}+c_{i}^{4}+c_{i}^{6}} /\left(c_{i}^{4}-1\right)$.

\section{Acknowledgements}

We are very thankful Camille Bonvin, Chiara Caprini, Sergei Dubovsky, Ruth Durrer, Valery Frolov, Robert Helling, Mattew Kleban, Lev Kofman, Stefano Liberati, Alan Rendall, Sergei Sibiryakov, Ilya Shapiro, Alexey Starobinsky, Leonard Susskind, Matt Visser, and especially Sergei Winitzki for very useful discussions. E.B. thanks Alexander von Humboldt foundation and INFN for support. 


\section{References}

[1] M. Born, L. Infeld, Proc.Roy.Soc.Lond.A144:425-451,(1934).

[2] C. Armendariz-Picon, T. Damour, V. Mukhanov, Phys.Lett. B458, 209-218 (1999) [hep-th/9904075]; C. Armendariz-Picon, V. Mukhanov, P. J. Steinhardt, Phys.Rev.Lett.85,4438-4441 (2000) [astro-ph/0004134]; C. Armendariz-Picon, V. Mukhanov, Paul J. Steinhardt, Phys. Rev. D63:103510 (2001) [astro-ph/0006373].

[3] S. Dubovsky, S. Sibiryakov, Phys.Lett.B638:509-514 (2006), [hep-th/0603158].

[4] C. Bonvin, C. Caprini, R. Durrer, Phys.Rev.Lett.97:081303 (2006), [astro-ph/0606584].

[5] J.U. Kang, V. Vanchurin, S. Winitzki, e-Print: arXiv:0706.3994 [gr-qc].

[6] J. Garriga, V. F. Mukhanov, Phys.Lett.B458, 219-225,(1999) [hep-th/9904176].

[7] V. Mukhanov, A. Vikman, (2006) JCAP 0602:004, [astro-ph/0512066]; A. Vikman astro-ph/0606033.

[8] C. Eling, T. Jacobson Class.Quant.Grav.23:5643-5660,2006, e-Print: gr-qc/0604088.

[9] E. Babichev, V. Mukhanov, A. Vikman, arXiv:0708.0561 [hep-th].

[10] E. Babichev, V. Mukhanov, A. Vikman, JHEP 0609,061 (2006) [hep-th/0604075].

[11] D. Blas, C. Deffayet, J. Garriga, Class. and Quant. Grav. 23 (2006) 1697 [hep-th/0508163].

[12] S. Mukohyama, Phys.Rev.D71, 104019 (2005) [hep-th/0502189].

[13] E. Babichev, V. Dokuchaev, Yu. Eroshenko, Phys.Rev.Lett. 93, 021102 (2004) [gr-qc/0402089]; Zh.Eksp.Teor.Fiz.100, 597, (2005) [astro-ph/0505618]

[14] W.G. Unruh, Phys.Rev.Lett.46:1351-1353,(1981).

[15] A. Adams, N. Arkani-Hamed, S. Dubovsky, A. Nicolis, R. Rattazzi,JHEP 0610:014,(2006), [hep-th/0602178].

[16] C. Armendariz-Picon, E. A. Lim, JCAP 0508:007, (2005),[astro-ph/0505207].

[17] A. D. Rendall, Class.Quant.Grav. 23, 1557-1570 (2006), [gr-qc/0511158].

[18] Y. Aharonov, A. Komar, L. Susskind, Phys.Rev.182:1400-1403,(1969).

[19] Jean-Philippe Bruneton, gr-qc/0607055, hep-th/0612113.

[20] W. B. Bonnor, Int. J. Mod. Phys. D12:1705-1708, (2003), [gr-qc/0211051]; Class. Quant. Grav.19:5951-5957, (2002); Amos Ori, gr-qc/0701024; J.Richard Gott, Phys. Rev. Lett.66:1126-1129, (1991).

[21] S. W. Hawking, Phys. Rev. D 46, 603 - 611 (1992).

[22] S. W. Hawking, G. F. R. Ellis, The large scale structure of space-time, Cambridge, 1973.

[23] R. M. Wald, General relativity, The Univ. of Chicago Press, 1984. 